\title{
LA SENSIBILIDAD INTERCULTURAL EN RELACIÓN CON LAS ACTITUDES DE ACULTURACIÓN Y PREJUICIO EN INMIGRANTES Y SOCIEDAD DE ACOGIDA. Un estudio de caso
}

\author{
INTERCULTURAL SENSITIVITY \\ REGARDING ACCULTURATION \\ ATTITUDES AND PREJUDICE IN \\ IMMIGRANTS AND THEIR HOST \\ SOCIETY. A case study
}

\author{
Antonio González LóPez \\ agonzalez@cop.es \\ María Paz Ramírez López \\ Universidad Pontificia Comillas, España. \\ pazramirezlopez@gmail.com
}

Universidad Nacional de Educación a Distancia, España.

Cómo citar este artículo / Citation: González López, A. y M. P. Ramírez López. 2016. "La sensibilidad intercultural en relación con las actitudes de aculturación y prejuicio en inmigrantes y sociedad de acogida. Un estudio de caso", Revista Internacional de Sociología, 74 (2): e034. Doi: http://dx.doi. org/10.3989/ris.2016.74.2.034

\footnotetext{
RESUMEN

El fenómeno de la globalización conlleva importantes implicaciones sociales que justifican la necesidad de estudiar la comunicación intercultural entre los grupos implicados en los procesos migratorios del siglo XXI. El objetivo principal de este trabajo es analizar la relación entre la sensibilidad intercultural y las actitudes de aculturación y prejuicio expresadas por una muestra de inmigrantes y de autóctonos formada por 255 participantes, en la ciudad de Cuenca (España). Los resultados obtenidos en este estudio de carácter exploratorio a través de análisis cuantitativos revelan que tanto los inmigrantes como los autóctonos optan por la asimilación como actitud de aculturación y que los inmigrantes manifiestan un mayor grado de sensibilidad intercultural. Los autóctonos que prefieren la integración muestran menos prejuicio, mientras que los inmigrantes que eligen la integración revelan mayor sensibilidad intercultural. Estos resultados se discuten en comparación con los encontrados en otros estudios con diferentes grupos sociales y culturales.
}

\section{Palabras Clave}

Comunicación intercultural; Diversidad cultural; Integración; Multiculturalismo; Relaciones interculturales.
Copyright: (C) 2016 CSIC. Este artículo de acceso abierto distribuido bajo los términos de la licencia Creative Commons Attribution-Non Commercial (by-cn) Spain 3.0.

Recibido: 10/02/2014. Aceptado: 10/02/2015.

Publicado on line: 18/05/2016

\section{Abstract}

The phenomenon of globalization has important social implications that justify the need to study the intercultural communication of groups involved in migratory processes in the 21st century. The main objective of this work is to analyze the relationship between the intercultural sensitivity and the attitudes of acculturation and prejudice expressed by a sample of immigrants and natives of the host society formed by 255 participants, in the city of Cuenca (Spain). Quantitative analysis revealed that both immigrants and natives choose assimilation as their attitude of acculturation and that immigrants showed a higher grade of intercultural sensitivity. Natives that prefer integration manifest less prejudice while immigrants that choose integration exhibit more intercultural sensitivity. These results are discussed in comparison to those found in other studies with different social and cultural groups.

\section{KEYWORDS}

Intercultural Communication; Cultural Diversity; Integration; Multiculturalism; Intercultural Relations. 


\section{INTRODUCCIÓN}

En el momento histórico actual determinados fenómenos característicos del siglo XXI, como la comunicación global, la diversidad o las migraciones, se constituyen en asuntos globales generadores de una cultura universal con importantes repercusiones en los contextos locales, donde en último término se manifiestan. Con respecto a las migraciones, en torno al $3 \%$ de la población mundial es considerada emigrante o persona que vive en otro país distinto al de su nacimiento como consecuencia de factores demográficos, desigualdades económicas, conflictos sociales y políticos o de la liberalización del comercio y de la mano de obra (International Organization for Migration 2009). En el caso concreto del contexto español, los estudios de población reflejan que más de cinco millones de personas nacidas en otros países viven dentro de nuestras fronteras, lo que supone que en torno al $10 \%$ de la población censada es inmigrante (Instituto Nacional de Estadística 2014); o que hace que nuestro país se haya convertido claramente en receptor de inmigrantes (Arango 2000). Esta situación de diversidad étnica y cultural está provocando importantes cambios socioculturales que obligan a entender la influencia del ambiente social sobre la convivencia y el bienestar psicológico necesarios para la integración entre las sociedades receptoras y los propios grupos de inmigrantes (Portes, Celaya, Vickstrom y Aparicio 2012).

En este sentido, las interacciones humanas transnacionales han puesto en primer plano términos como "multiculturalismo", "diversidad cultural" y "aculturación". En concreto, la "aculturación" ya fue definida en la primera mitad del siglo XX como "aquellos fenómenos que resultan cuando grupos de individuos con diferentes culturas entran en contacto continuo, con cambios posteriores en los patrones culturales originales de uno o ambos grupos" (Redfield, Linton y Herskovitz 1936: 149). De acuerdo a la importancia de este concepto, desde las ciencias sociales se han desarrollado numerosos modelos teóricos para evaluar el proceso de aculturación y los cambios en los valores e identidad cultural que se generan entre grupos inmigrantes y sociedad receptora (Berry 1980; Padilla 1980; Bourhis, Moïse, Perreault y Sénecal 1997; Piontkowski, Rohmann y Florack 2002; Navas et al. 2005). De esta forma, la aculturación ha sido analizada por los distintos modelos teóricos en relación con el ajuste sociocultural y la adaptación psicológica a un nuevo contexto sociocultural (Ward y Kennedy 1994) y también con variables psicosociales implicadas en las relaciones intergrupales (Padilla y Perez 2003).

En concreto, el Modelo de Aculturación de Berry (1980) plantea que la aculturación sucede a través de un proceso caracterizado por el contacto, el conflicto y la adaptación. A lo largo de este proceso adaptativo se dan cuatro respuestas u opciones de aculturación, según el valor que los grupos minoritarios conceden o no a mantener su propia herencia cultural y al valor otorgado a relacionarse o no con la cultura de acogida. Estas respuestas adaptativas son la "integración", la "asimilación", la "separación" y la "marginación". La "integración" se refiere al mantenimiento de la cultura de origen a la vez que se produce una apertura a la sociedad de acogida. La "asimilación" se da cuando se abandona la cultura de origen y se dan relaciones con la sociedad de acogida. La "separación" sucede cuando se mantiene la identidad cultural de origen y no hay relación con la sociedad receptora, y la "marginación" ocurre cuando los inmigrantes abandonan su cultura de origen y además no establecen contacto con la sociedad de acogida. A este respecto, numerosos trabajos concluyen que la integración es la estrategia adaptativa preferida por los inmigrantes y la que se relaciona con mayores niveles de salud mental y con mejores actitudes intergrupales (Berry 1997; Pfafferott y Brown 2006). Sin embargo, este modelo de categorías de aculturación ha sido criticado por asumir que las cuatro respuestas de aculturación tienen la misma validez, de tal forma que se ha llegado a plantear la posible existencia de opciones de aculturación propias de cada individuo (Bourhis et al. 1997; Rudmin 2009).

Por su parte, el Modelo Ampliado de Aculturación Relativa (Navas et al. 2005) hace referencia a dos planos de aculturación. El plano real, referido a las estrategias de aculturación que eligen los inmigrantes y que los autóctonos perciben que los inmigrantes ponen en práctica; y el plano ideal, referente a las actitudes u opciones de aculturación preferidas por inmigrantes y que los autóctonos prefieren para ellos. Desde este modelo, se concluye que el proceso adaptativo es complejo y relativo, pues se dan diferentes estrategias y distintas actitudes dependiendo de que la interacción entre inmigrantes y autóctonos se produzca en unos u otros ámbitos de la realidad sociocultural. Estos ámbitos son el "político" -sistema político y de gobierno-, "laboral" -procedimientos del trabajo-, "económico" -hábitos de consumo y economía familiar-, "social" -relaciones sociales y amistades-, "familiar", "religioso" y, finalmente, el ámbito de las "formas de pensar", donde se encuadran los principios y los valores (Navas et al. 2005). Determinados trabajos de investigación concluyen que hay una escasa correspondencia entre las opciones de aculturación de los inmigrantes y la percepción de dichas elecciones por parte de los autóctonos, debido a que estas percepciones suelen estar sesgados por otras actitudes negativas hacia el grupo inmigrante (van Oudenhoven, Prins y Buunk 1998; Zagefka y Brown 2002). En concreto, la discriminación o el prejuicio, entendido como una actitud negativa hacia un grupo social o hacia sus miembros (Allport 1954; Brown 1995), se constituye en sesgos que condicionan las preferencias y percepciones del proceso de aculturación de los grupos de inmigrantes por parte de la sociedad de acogida (Briones, Verkuyten, Cosano y Tabernero 2012). 
A este respecto, algunos trabajos han encontrado que, tanto en poblaciones mayoritarias como en los grupos minoritarios o de inmigrantes, las puntuaciones más bajas en prejuicio hacia el exogrupo se relacionan de forma positiva con la preferencia por la integración; y las puntuaciones más altas, con la preferencia por la marginación (Navas et al. 2006; Zick, Wagner, van Dick y Petzel 2001). En la misma línea, la concordancia o discordancia entre la sociedad de acogida y los grupos de inmigrantes en las preferencias sobre las opciones de aculturación influiría en sus relaciones intergrupales, en los posibles conflictos, e incluso en la elección de unas u otras opciones de aculturación por parte del grupo minoritario (Piontkowski, Rohmann y Florack 2002; Kosic, Mannetti y Sam 2005). Por todo ello, para entender el proceso de adaptación social y la convivencia entre grupos culturales, es necesario considerar la influencia que las actitudes del grupo mayoritario ejercen sobre la forma en que las minorías culturales afrontan su proceso de aculturación. En este punto, cabe destacar las aportaciones del interculturalismo entendido como proceso de interacción y adaptación, a través de un intercambio social entre los diferentes grupos, basado en la negociación y la resolución de los conflictos, que desencadena cambios de la estructura social beneficiosos para los grupos culturales (Rodríguez-García, 2010).

Siguiendo este objetivo de generar actitudes de entendimiento e interacción entre grupos diferentes, hay que considerar el concepto de "competencia comunicativa intercultural" entendida como el "conjunto de conocimientos, destrezas y actitudes interculturales necesarias para comunicarse y convivir de forma apropiada en sociedades caracterizadas por la diversidad cultural" (Gudykunst, Wiseman y Hammer 1977; Ruben y Kealy 1979; Chen 1990). A este respecto, el Modelo de Desarrollo de la Sensibilidad Intercultural (Bennett 1993) plantea que la convivencia entre culturas se basa en la sensibilidad intercultural definida como un cambio afectivo, cognitivo y conductual que iría desde posiciones etnocéntricas a posiciones etnorrelativas de reconocimiento y apreciación de las diferencias culturales. Este modelo asume que, conforme se experimentan diferencias culturales más complejas, se incrementa la competencia para las relaciones interculturales a través de un proceso de transformación basado en seis fases o etapas: 1. Negación, donde se niegan las diferencias culturales importantes y se concibe la propia cultura como la única verdadera; 2. Defensa, cuando las diferencias culturales se viven como amenazantes y se considera la propia cultura como la única viable; 3 . Minimización, donde para proteger la propia cosmovisión se resaltan las similitudes culturales enmascarando el reconocimiento y apreciación de las diferencias; 4. Aceptación, cuando se reconocen y aprecian las diferencias y similitudes de la propia y de otras culturas; 5 . Adaptación, sucede cuando la persona puede cambiar su perspectiva cul- tural, mostrar empatía con las diferencias culturales y modificar los afectos e identidad cultural sobre la base de las diferencias culturales; y 6 . Integración, cuando la propia experiencia del yo incluye las diferentes visiones culturales.

Por su parte, el Modelo de Competencia Comunicativa Intercultural (Chen y Starosta 1996) entiende la convivencia entre culturas por medio de la construcción de un multiculturalismo sustentado en la integración de las diferencias culturales y en la interacción intercultural respetuosa. Según este modelo, la comunicación intercultural consta de tres componentes: 1. La sensibilidad intercultural o aspecto afectivo, referida al deseo emocional de reconocer, aceptar y apreciar las diferencias culturales en situaciones de interacción intercultural; 2. La conciencia intercultural o dimensión cognitiva, relativa al proceso de entendimiento de las convenciones culturales y de las similitudes-diferencias respecto a otra cultura; y 3. La competencia intercultural o aspecto conductual, referida a las habilidades de consecución de las metas comunicativas al interactuar con personas de otras culturas. De esta forma, la sensibilidad intercultural en cuanto componente afectivo se define como "la capacidad personal para desarrollar una emoción positiva hacia el entendimiento y aprecio de las diferencias culturales".

Siguiendo la línea teórica arriba apuntada, el principal objetivo de este trabajo consiste en estudiar la sensibilidad intercultural y las orientaciones de aculturación en una muestra de la población inmigrante y de la población autóctona en la ciudad de Cuenca. Se identifican las actitudes multiculturales y de aculturación de ambas muestras y se analizan sus relaciones con la sensibilidad intercultural y con el prejuicio, entendido como una actitud negativa hacia un grupo social o hacia sus miembros. De tal forma que las principales hipótesis que guían esta investigación son:

1. Los inmigrantes prefieren las actitudes de integración en el proceso de adaptación sociocultural, mientras que la población autóctona prefiere que los inmigrantes adopten una respuesta de asimilación.

2. Los inmigrantes muestran mayores niveles de sensibilidad intercultural que la población autóctona.

3. Los autóctonos que prefieren la integración de los inmigrantes muestran menos actitud prejuiciosa que aquellos que prefieren otras opciones de aculturación.

4. Los inmigrantes y autóctonos que se decantan por las actitudes de integración manifiestan mayores niveles de sensibilidad intercultural que los que optan por el resto de actitudes de aculturación.

De esta forma, este trabajo de carácter exploratorio se convierte en un primer intento hacia un modelo de integración sociocultural de la población inmigrante en un contexto local específico, poniendo en relación una serie de variables como son las respuestas 
de aculturación, el prejuicio como actitud social de discriminación y la sensibilidad intercultural en cuanto actitud hacia las interacciones culturales y ante la diversidad étnica. Esta línea de estudio es coincidente con la investigación en aculturación que considera la integración constructiva como un resultado de la competencia intercultural y del etnorelativismo (Bennet, 1993; Boski 2008).

\section{Método}

\section{Participantes}

Un total de 225 personas participaron en este trabajo. La muestra se distribuyó en 105 inmigrantes y 120 autóctonos de la ciudad de Cuenca. El trabajo de campo se llevó a cabo en los meses de diciembre de 2012 y enero de 2013, durante los cuales una encuestadora entrenada al efecto visitó las dependencias de Cáritas, Cruz Roja y Asociaciones de Inmigrantes en Cuenca, donde solicitó a inmigrantes mayores de edad su participación en este estudio de investigación, haciéndoles entrega del cuestionario con las instrucciones precisas parar ser cumplimentado en ese mismo momento de forma individual y anónima. Por otro lado, otra encuestadora durante el mismo tiempo visitó centros de trabajo públicos y privados donde pidió a los presentes su participación en este estudio de investigación a través de la cumplimentación del cuestionario con las instrucciones precisas para ser rellenado. En ambos casos, por tanto se siguió un muestreo no probabilístico y todos los sujetos a los que se hizo entrega del cuestionario lo cumplimentaron y entregaron en ese mismo momento.

Con respecto a la muestra de inmigrantes, 44 fueron hombres y 61 mujeres, siendo la media de edad 36,34 años $(D T=10,42)$ y su media de tiempo de estancia en España de 8,08 años (DT=4,03). Por lo que respecta a la muestra de autóctonos, 55 fueron hombres y 65 mujeres, siendo la media de edad 41,14 años (DT=12,75). En las Tablas 1 y 2 se refleja la distribución en porcentajes de la muestra de inmigrantes y autóctonos respectivamente, en función de las características sociodemográficas consideradas.

En el cuestionario se preguntó también a los inmigrantes sobre su identidad, obteniéndose unos resultados porcentuales de un 21,2 \% que manifestó sentirse de su país de origen frente al $15.4 \%$ que dijo sentirse de España, mientras que un total de un $62,5 \%$ manifestó tener una identidad compartida, y tan solo un $1 \%$ dijo no sentirse de ningún país. Con respecto a una cuestión relativa a los amigos o personas con las que más se relacionan, las respuestas porcentuales fueron muy similares a las anteriores puesto que un $21,2 \%$ respondió que se relaciona solo con personas de su propio país y un $62,5 \%$ que se relaciona con personas de ambos países, mientras que un $11,5 \%$ dijo relacionarse solo con españoles y un $4,8 \%$ solo con personas de terceros países.
Tabla 1.

Descriptivos sociodemográficos de la muestra de inmigrantes

\begin{tabular}{|c|c|c|}
\hline VARIABLE & GRUPOS & PORCENTAJE \\
\hline \multirow{3}{*}{ Lugar de procedencia } & Este de Europa & 19,8 \\
\hline & Norte de África & 27,4 \\
\hline & América Latina & 52,8 \\
\hline \multirow{3}{*}{ Tiempo en España } & 1-5 años & 26 \\
\hline & 6-10 años & 49 \\
\hline & + 11 años & 25 \\
\hline \multirow{2}{*}{ Género } & Hombre & 41.9 \\
\hline & Mujer & 58,1 \\
\hline \multirow{4}{*}{ Edad } & $18-25$ & 16,3 \\
\hline & $26-33$ & 25 \\
\hline & $34-41$ & 34,6 \\
\hline & +42 & 24 \\
\hline \multirow{4}{*}{ Situación laboral } & Estudiante & 4.8 \\
\hline & Trabajador & 25,7 \\
\hline & Desempleado & 67,6 \\
\hline & Jubilado & 1,9 \\
\hline \multirow{4}{*}{ Nivel de estudios } & Sin estudios & 3,8 \\
\hline & Primarios & 13,2 \\
\hline & Medios & 63,2 \\
\hline & Superiores & 19,8 \\
\hline \multirow{4}{*}{ Estado civil } & Soltero & 30,2 \\
\hline & Casado/pareja & 62,3 \\
\hline & Viudo & 1,9 \\
\hline & Separado & 5,7 \\
\hline
\end{tabular}

Fuente: Elaboración propia.

\section{Instrumento}

Para conseguir los objetivos propuestos, se construyó un doble cuestionario dirigido tanto a la población general como a inmigrantes. En el caso de los autóctonos, este instrumento estuvo formado por una serie de preguntas divididas en los siguientes apartados según su orden de aparición en el propio cuestionario:

1. Características sociodemográficas. Género, edad, estudios realizados, estado civil, situación laboral, creencias políticas y frecuencia de relación con personas de otros países.

2. Actitudes de aculturación. Se creó esta nueva variable basada en las dimensiones del Modelo de Berry (1997) sobre la importancia concedida, o no, a la conservación de la identidad cultural de origen, y sobre la importancia de relacionarse, o no, con la sociedad de acogida. De esta forma, se elaboró una medida dicotómica para comprobar las preferencias sobre aculturación de los autóctonos para los inmigrantes por medio de dos cuestiones formadas por doble opción de respuesta donde solo se podía elegir una de las opciones. En un caso, se preguntó a los au- 
Tabla 2.

Descriptivos sociodemográficos de la muestra de autóctonos

\begin{tabular}{|c|c|c|}
\hline VARIABLE & GRUPOS & PORCENTAJE \\
\hline \multirow{4}{*}{ Situación laboral } & Estudiante & 7,5 \\
\hline & Trabajador & 72,5 \\
\hline & Desempleado & 15,8 \\
\hline & Jubilado & 4,2 \\
\hline \multirow{2}{*}{ Género } & Mujer & 54,2 \\
\hline & Hombre & 45,8 \\
\hline \multirow{3}{*}{ Edad } & $18-35$ & 35,7 \\
\hline & $36-41$ & 15,2 \\
\hline & +42 & 49,1 \\
\hline \multirow{4}{*}{ Estudios } & Sin estudios & 0,8 \\
\hline & Primarios & 4,2 \\
\hline & Medios & 34,2 \\
\hline & Superiores & 60,8 \\
\hline \multirow{4}{*}{ Creencias políticas } & Hacia derecha & 27,5 \\
\hline & Hacia izquierda & 35,8 \\
\hline & Ni una ni otra & 22,5 \\
\hline & Me es indiferente & 14,2 \\
\hline \multirow{4}{*}{ Estado civil } & Soltero & 36,1 \\
\hline & Casado/con pareja & 53,8 \\
\hline & Viudo & 1,7 \\
\hline & Separado & 8,4 \\
\hline \multirow{4}{*}{$\begin{array}{l}\text { Relación con } \\
\text { personas } \\
\text { de otros países }\end{array}$} & Mucho & 17,5 \\
\hline & Bastante & 35 \\
\hline & Poco & 40,8 \\
\hline & Nada & 6,7 \\
\hline
\end{tabular}

Fuente: Elaboración propia.

tóctonos si prefieren que los inmigrantes vivan según la forma de vida y costumbres de su país, o bien, según la forma de vida y costumbres de los españoles. En otro, si prefieren que se relacionen y convivan con los españoles, o bien, que se relacionen y convivan con sus paisanos. Según la combinación de las opciones de respuesta de ambas cuestiones se asigna los sujetos a las diferentes actitudes de aculturación; en concreto: si prefieren que vivan según sus propias costumbres y se relacionen con españoles se asignan a la integración, si prefieren que vivan según las costumbres españolas y se relacionen con españoles se asignan a la asimilación, si prefieren que vivan según sus costumbres de origen y solo se relacionen entre ellos mismos se asigna a la separación y, finalmente, si prefieren que vivan según las costumbres españolas y solo se relacionen entre ellos, a la marginación.

3. Test de multiculturalismo. Consta de 4 cuestiones obtenidas de las escalas de actitudes étnicas y multiculturales de Berry y Kalim (1995). El objetivo de esta escala era medir el grado de acuerdo o desacuerdo con la idea de una socie- dad multicultural a través de las siguientes cuestiones: "Es beneficioso que haya diferentes grupos culturales conviviendo en un mismo país", "Me siento aceptado por los otros grupos culturales que me rodean", "Es una idea aceptable que personas de diferentes culturas se casen entre ellas", y, "La diversidad cultural es una característica deseable para la sociedad mayoritaria". El formato fue de respuesta tipo Likert de 5 puntos desde 'Muy de acuerdo' a 'Muy en desacuerdo'.

4. Escala de sensibilidad intercultural (Chen y Starosta 2000). Formada por un total de 24 ítems en formato tipo Likert de 5 puntos desde 'Muy de acuerdo' a 'Muy en desacuerdo', encaminados a valorar la implicación, respeto, seguridad, disfrute y sensibilidad en las interacciones interculturales.

5. Test de prejuicio: Con el objetivo de comprobar la existencia de actitudes de prejuicio entre los participantes autóctonos hacia los inmigrantes, se incluyó el Test de Actitud Prejuiciosa (TAP) de Rojas, Navas, Lozano y Pérez (2011). Según estos autores, el TAP es una escala con adecuadas propiedades psicométricas que permite hacer comparaciones entre grupos y medir las peculiaridades de las actitudes sociales de prejuicio basadas en cogniciones o estereotipos, en emociones o afectos y en intenciones de conducta o distancia social preferida. Sin embargo, en este trabajo se eliminó la dimensión cognitiva por considerar, de la misma forma que Brown (1995), que el prejuicio puede ser entendido desde las dimensiones cognitiva, emocional y conductual, solas o combinadas entre sí. De esta forma, el test estuvo formado por 7 cuestiones de afecto y una cuestión sobre intención de conducta; en concreto 3 ítems de emociones positivas ('admiración', 'simpatía' y 'respeto') y 4 ítems de emociones negativas sutiles ('desconfianza', 'incomodidad', 'inseguridad' e 'indiferencia'). Los participantes indicaron el grado en el que habían sentido cada una de esas emociones hacia los inmigrantes, para ello se utilizó una escala de frecuencia desde ' 1 ' (nada) a ' 5 ' (mucho).

Con respecto al cuestionario dirigido a inmigrantes, estuvo formado por una serie de preguntas divididas en varios apartados:

1. Características sociodemográficas. Género, edad, país de nacimiento, estudios realizados, estado civil, tiempo en España, situación laboral, sentimiento de identidad y nacionalidad de amigos y conocidos.

2. Actitudes de aculturación. Idéntico al de autóctonos pero preguntando por su propia preferencia de forma de vida y de relacionarse.

3. Test de multiculturalismo. La misma escala sobre actitudes étnicas y multiculturales que para autóctonos. 
4. Índice de Aculturación de Vancouver (VIA) (Ryder, Aldem y Paulhus 2000). Consta de 20 ítems en formato de respuesta tipo Likert de 5 puntos desde 'Muy de acuerdo' a 'Muy en desacuerdo'. Este índice contempla un modelo bidimensional de aculturación a través de la medición de valores, relaciones sociales y adherencia a las tradiciones, tanto de la cultura de origen como de la de acogida.

5. Escala de sensibilidad intercultural (Chen y Starosta 2000).

\section{Resultados}

\section{Actitudes de aculturación en inmigrantes y au- tóctonos}

Conforme se ha mencionado, se comprobaron las actitudes de aculturación de inmigrantes y población autóctona asignando a los participantes a una de las cuatro orientaciones de aculturación referidas. Los porcentajes de respuesta de las muestras respecto a estas cuestiones pueden verse en la Figura 1.

Como puede observarse, un $51 \%$ de los inmigrantes de la muestra en la ciudad de Cuenca se decantan por la asimilación como opción de aculturación en su proceso de adaptación a la sociedad receptora. La integración se constituye en la segunda opción elegida, mientras que la marginación y separación son las actitudes menos elegidas en el proceso de adaptación. Según estos resultados, una amplia mayoría de inmigrantes manifiestan actitudes de asimilación en su proceso de aculturación.

Con respecto a los porcentajes de respuesta de la población autóctona de Cuenca, como puede verse, casi un $70 \%$ de la muestra se decanta por la asimilación como opción de aculturación preferida para que los inmigrantes se adapten a la sociedad receptora. La integración se constituye en la segunda opción, mientras que la marginación y separación son actitudes apenas elegidas.

\section{Sensibilidad intercultural, aculturación, multicul- turalismo y prejuicio}

En un primer momento y con el objetivo de comprobar el funcionamiento de las diferentes escalas utilizadas en este trabajo, se llevaron a cabo análisis de fiabilidad por medio del coeficiente $\alpha$ de Cronbach y se obtuvo la media (M) y la desviación típica (DT) para cada una de ellas. Estos valores pueden contemplarse en la Tabla 3.

Posteriormente, con el objetivo de obtener un primer acercamiento a las posibles relaciones existentes entre las variables consideradas, se realizaron análisis de correlación de Pearson. En las Tablas 4 y 5 pueden observarse las matrices de correlación obtenidas por la muestra de inmigrantes y autóctonos en las diferentes escalas. Conforme puede observarse en la Tabla 4, se obtiene una correlación positiva

Figura 1.

Actitudes de aculturación en la muestra de autóctonos e inmigrantes

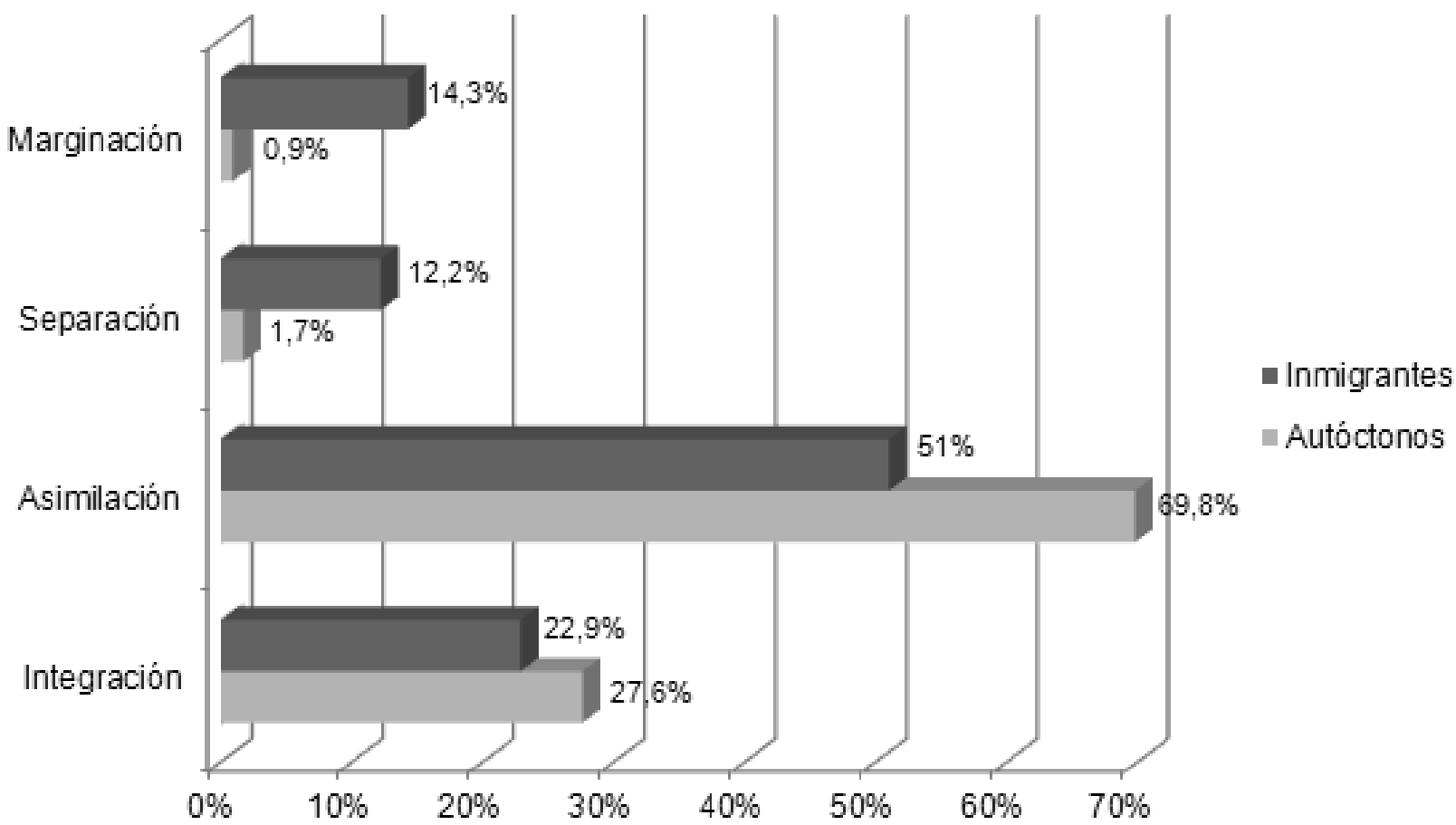

Fuente: Elaboración propia. 
Tabla 3.

Fiabilidad, media y desviación típica de las escalas en inmigrantes y autóctonos

\begin{tabular}{|c|c|c|c|c|c|c|}
\hline \multirow{2}{*}{ Escalas } & \multicolumn{2}{|c|}{$\alpha$ de Cronbach } & \multicolumn{2}{c|}{ Media Desviación Típica } \\
\cline { 2 - 7 } & I & A & I & A & I & A \\
\hline Multiculturalismo & 0,67 & 0,71 & 14,13 & 15,16 & 3,61 & 2,81 \\
\hline Sensibilidad Intercultural & 0,78 & 0,86 & 87,81 & 91 & 11,65 & 10,02 \\
\hline VIA cultura Origen & 0,86 & - & 37,62 & - & 7,98 & - \\
\hline VIA cultura Acogida & 0,86 & - & 35,91 & - & 7,53 & - \\
\hline Prejuicio & - & 0,81 & - & 30,03 & - & 5,13 \\
\hline
\end{tabular}

Fuente: Elaboración propia.

entre la sensibilidad intercultural y el multiculturalismo en la muestra de inmigrantes; y también de estas variables con la aculturación medida a través de la escala VIA (Índice de Aculturación de Vancouver), principalmente entre multiculturalismo y la preferencia por la cultura de acogida. Es decir, aquellos inmigrantes que se orientan en mayor medida hacia la cultura de acogida mostrarían mayor sensibilidad intercultural y tendencia hacia el multiculturalismo. De esta forma, la sensibilidad intercultural, el multiculturalismo y la aculturación correlacionan de una forma positiva para el caso de los inmigrantes. De este resultado se desprende que aquellos inmigrantes con actitudes biculturales mostrarían una clara sensibilidad intercultural y una tendencia al multiculturalismo entendido como actitud favorable hacia la convivencia entre culturas, sugiriendo una clara receptividad de los inmigrantes a mantener valores, relaciones y tradiciones de ambas culturas en su proceso de aculturación, lo que avala por tanto un modelo bicultural de aculturación.

Con respecto a la muestra de autóctonos, en la Tabla 5 podemos observar las relaciones entre las variables consideradas. Cabe destacar que, en esta muestra, la sensibilidad intercultural no se relacionó con ninguna de las variables restantes. El multiculturalismo se relacionó con el prejuicio, de tal forma que las personas menos prejuiciosas se muestran más multiculturales.

Con el objetivo de ahondar en las posibles relaciones existentes entre las diferentes variables contempladas en este trabajo, se realizaron otros análisis bivariantes. En concreto, y con la muestra de inmigrantes, se realizaron ANOVAS contemplando las principales actitudes de aculturación como variables independientes, y las diferentes escalas como variables dependientes. Hay que recordar, como se ha mencionado en el apartado de instrumento, que las actitudes de aculturación en cuanto variable independiente se generan a partir del resultado obtenido de las variables dicotómicas obtenidas tras la combinación de respuestas a las dos
Tabla 4.

Matriz de correlaciones en la muestra de inmigrantes $(\mathrm{N}=105)$

\begin{tabular}{|l|c|c|c|c|}
\hline Escalas e ítems & 1 & 2 & 3 & 4 \\
\hline 1. Multiculturalismo & - & & & \\
\hline 2. Sensibilidad Intercultural &, $63^{* *}$ & - & & \\
\hline 3. VIA Origen &, $35^{* *}$ &, $50^{* *}$ & - & \\
\hline 4.VIA Acogida &, $56^{* *}$ &, $24^{*}$ &, $26^{*}$ & - \\
\hline
\end{tabular}

$P<0,01$.

Fuente: Elaboración propia.

Tabla 5.

Matriz de correlaciones en la muestra de autóctonos $(\mathrm{N}=120)$

\begin{tabular}{|l|c|c|c|}
\hline Escalas e ítems & 1 & 2 & 3 \\
\hline 1. Multiculturalismo & - & & \\
\hline 2. Prejuicio &, $35^{\star *}$ & - & \\
\hline 3. Sensibilidad Intercultural &, 07 &, 05 & - \\
\hline
\end{tabular}

$P<0,01$.

Fuente: Elaboración propia.

preguntas sobre la importancia concedida o no a la conservación de la identidad cultural de origen y a la importancia de relacionarse o no con la sociedad de acogida. Dependiendo de las respuestas obtenidas, se asignó a los participantes a una de las cuatro actitudes de aculturación mencionadas.

Se encontraron diferencias significativas en la escala de sensibilidad intercultural $(F=4,71 ; g l=2 ; p<, 05)$, mostrando la prueba de comparación de medias de Scheffé que se daban diferencias estadísticamente significativas entre el grupo de integración $(M=93,33$; 
DT=12,5) y el grupo de separación $(M=77,56$; $\mathrm{DT}=8,6)$. De esta forma, los inmigrantes que prefieren la integración en su proceso de aculturación mostraron más sensibilidad intercultural que aquellos que prefieren la separación como opción de aculturación. También se encontraron diferencias significativas en la opción 'cultura de origen' ( $F=5,92 ; \mathrm{gl}=2 ; \mathrm{p}<, 005)$ del Índice de Aculturación de Vancouver, mostrando la prueba de Scheffé diferencias estadísticamente significativas de los grupos de integración $(M=40,12$; $D .=8,7)$ y separación $(M=40,90 ; D T=6,1)$ frente al grupo de asimilación (M=33,93; DT=7,3). De esta forma, los inmigrantes que optaron por la integración y la separación en su proceso de aculturación se mostraron más identificados con su cultura de origen que los que optaron por la asimilación.

Para el caso de los autóctonos, se llevó a cabo un análisis $t$-test considerando las actitudes de integración y asimilación, que fueron las mayoritariamente elegidas, como variables independientes, y las diferentes escalas como variables dependientes. Los resultados obtenidos mostraron diferencias significativas en la escala de multiculturalismo ( $\mathrm{t}=3.23$; $\mathrm{p}<.005)$. La prueba Scheffé de contraste de medias mostró que los autóctonos que optaron por la integración $(\mathrm{M}=16.58$; $\mathrm{DT}=2.2)$ como opción preferida de aculturación para los inmigrantes, se mostraron más favorables al multiculturalismo que los que eligieron la asimilación ( $M=14,71 ; D T=2,9)$. También se encontraron diferencias significativas en la escala de prejuicio ( $t=3,83 ; \mathrm{gl}=107 ; \mathrm{p}<, 001)$, de tal forma que el grupo que optó por la integración $(M=33,03$; $D T=3,7)$ manifestó menos prejuicio que el grupo que eligió la asimilación (M=29,16; DT=5).

Finalmente, con el objetivo de conocer las posibles diferencias entre las dos muestras utilizadas en este trabajo en relación a las diferentes escalas utilizadas, se llevó a cabo un análisis $t$-test para muestras independientes. Por medio de esta prueba se encontraron diferencias significativas en las puntuaciones obtenidas en la escala de sensibilidad intercultural $(t=12,14 ; p<, 001)$. Según las puntuaciones medias obtenidas por ambas muestras, los inmigrantes mostraron mayor grado de sensibilidad intercultural $(M=87,81 ; D T=11,6)$ que los autóctonos $(M=71,94$; $\mathrm{DT}=5,5)$. De este modo, se concluye que los inmigrantes manifiestan un mayor grado de sensibilidad intercultural que los autóctonos posiblemente debido a su necesidad de interacción con la cultura de acogida que les obliga a desarrollar la sensibilidad en las relaciones interculturales.

\section{Discusión}

Este trabajo representa un estudio sobre aculturación y sensibilidad intercultural en inmigrantes y autóctonos. El principal objetivo ha consistido en estudiar las relaciones existentes entre la sensibilidad intercultural, entendida como dimensión afectiva de la competencia comunicativa intercultural, y las actitudes de aculturación en la población autóctona e inmigrante de la ciudad de Cuenca, con base en los modelos de sensibilidad intercultural de Chen y Starosta (1996) y de aculturación de Berry (1980).

Los resultados encontrados muestran que los inmigrantes prefieren la asimilación como opción de aculturación en su proceso de adaptación a la sociedad receptora, siendo la integración la segunda actitud elegida. Por su parte, la población autóctona también eligió la asimilación y la integración como las principales opciones de aculturación preferidas para los inmigrantes en su proceso de adaptación a la sociedad de acogida. En este sentido no ha podido ser confirmada la primera hipótesis planteada, relativa a que los inmigrantes prefieren las actitudes de integración, mientras que la población autóctona prefiere la actitud de asimilación. A este respecto, es relevante que también otros trabajos encuentran un alto grado de concordancia entre la población inmigrante y autóctona en sus actitudes de aculturación que a su vez se relaciona con bajos niveles de conflicto en las relaciones intergrupales y con menor expresión de prejuicio (Navas, Rojas y García 2011), si bien este aspecto no fue específicamente analizado en el presente trabajo. Sin embargo, los resultados obtenidos revelan que aquellos autóctonos que prefieren la integración manifiestan menos prejuicio que aquellos otros que optan por la asimilación, confirmando de este modo la hipótesis en la que se planteaba que los autóctonos e inmigrantes que optan por la opción de integración muestran menor nivel de prejuicio; y coincidiendo así con estudios que encuentran hallazgos muy similares (Navas et al. 2006; Zick et al. 2001).

Con respecto a la sensibilidad intercultural, considerada como dimensión afectiva de la competencia comunicativa intercultural, los resultados nos muestran que los inmigrantes que prefieren la integración puntúan más alto en sensibilidad intercultural frente a los que eligen la separación. A su vez, los inmigrantes manifiestan mayor grado de sensibilidad intercultural que los autóctonos, lo que apuntaría a su mayor grado de implicación, respeto y sensibilidad en las interacciones interculturales. De esta forma, se confirman las hipótesis planteadas al respecto sobre la mayor sensibilidad intercultural en inmigrantes y también en aquellos que optan por la integración frente a otras opciones de aculturación.

Por otro lado, también entre los inmigrantes se encontraron altas correlaciones entre la sensibilidad y la identificación con la cultura de origen y con la de acogida, por ello la sensibilidad intercultural se mostró implicada en un posible modelo de competencia bicultural y de integración constructiva (Bennet 1993; Boski 2008). De la misma forma, la sensibilidad intercultural se relacionó con el multiculturalismo en la muestra de inmigrantes, lo que podría estar indican- 
do el respeto y sensibilidad de los inmigrantes hacia otras culturas en general y hacia la cultura de acogida en particular. Estos resultados son coincidentes con los obtenidos en otros trabajos que contemplan la sensibilidad intercultural y el multiculturalismo como aspectos claramente implicados en la promoción de la diversidad cultural y en la superación del etnocentrismo (Berry y Kalim 1995; Chen y Starosta 2004).

Los resultados encontrados en este trabajo de carácter exploratorio pueden considerarse como una primera contribución a este campo de estudio, poniendo de relieve la importancia futura de la dimensión aplicada de la sensibilidad intercultural, en cuanto competencia comunicativa intercultural basada en la implicación, respeto y disfrute de las relaciones culturales, como una importante variable para entender y fomentar la integración, la reducción de actitudes de discriminación y la superación del etnocentrismo; todo ello con el objetivo último de generar un modelo multicultural basado en la comprensión de los procesos de aculturación adecuados en la superación de conflictos, prejuicios y otras actitudes intergrupales negativas.

Finalmente, los resultados obtenidos en este trabajo deben ser considerados bajo algunas limitaciones metodológicas que hacen imposible su generalización y exigen cautela en su interpretación. Por un lado, en la medida en que las muestras utilizadas no son representativas de la población inmigrante ni tampoco de la población general de la ciudad, las conclusiones en ningún caso pueden generalizarse. En este sentido, cabe mencionar que no se conside-

\section{Referencias Bibliográficas}

Allport, G. W. 1954. The nature of prejudice. Reading, MA: Addison-Wesley.

Arango, J. 2000. "Becoming a Country of Immigration at the End of the Twentieth Century: The Case of Spain". Pp. 253-276 in El Dorado o Fortresss? Migration in Southern Europe, edited by R. King, G. Lazaridis, C. Tsardanidis. London: Palgrave MacMlllan.

Bennett, M. J. 1993. "Towards ethnorelativism: A developmental model of intercultural sensitivity". Pp. 21-71 in Education for the intercultural experience, 2nd ed. by R. M. Paige. Yarmouth, ME: Intercultural Press.

Berry, J. W. 1980. "Acculturation as varieties of adaptation". Pp. 9-25 in Acculturation: Theory, edited by A. M. Padilla. Boulder, CO: Westview.

Berry, J. W. 1997. "Immigration, acculturation and adaptation". Applied Psychology: An International Review 46:5-68. http://dx.doi.org/10.1111/j.1464-0597.1997.tb01087.x

Berry, J. W. and R. Kalim. 1995. "Multicultural and ethnic attitudes in Canada: An overview of the 1991 national survey". Canadian Journal of Behavioral Science 27:301320. http://dx.doi.org/10.1037/0008-400X.27.3.301

Bosky, P. 2008. "Five meanings of integration in acculturation research". International Journal of Intercultural Relations 32:142-153. http://dx.doi.org/10.1016/j.ijintrel.2008.01.005 ró apropiado desagregar las muestras utilizadas por grupos en función de sus variables sociodemográficas debido a su escaso tamaño en este trabajo. A pesar de ello, los datos obtenidos con ambas muestras permiten considerar este trabajo exploratorio como un primer acercamiento al tema de la sensibilidad intercultural y de la aculturación. Por otro lado, para la realización de esta investigación se elaboró un cuestionario específico integrado por una serie de escalas obtenidas expresamente para la consecución de los objetivos propuesto que, si bien en su conjunto han demostrado cierta validez, sin embargo necesitan ser mejoradas para su uso como instrumentos válidos y fiables en futuros trabajos de investigación. De la misma forma, con el objetivo de obtener resultados fiables y generalizables, será necesario probar estas escalas con nuevas muestras obtenidas con procedimientos muestrales que permitan acceder a inmigrantes menos vinculados con organizaciones o asociaciones y con muestras de autóctonos más representativas del conjunto de la población general, además de valorar posibles resultados según diferentes dominios sociales y ámbitos de vida, de tal forma que sea posible la comparación de los resultados con otros estudios sobre actitudes interculturales. La obtención de resultados generalizables y, consecuentemente la generación de modelos psicosociales de integración e interculturalidad aplicables a diversos contextos sociales y con diferentes grupos etnoculturales, permitirá entender las relaciones intergrupales en las comunidades locales donde se manifiestan las actitudes y los comportamientos entre grupos sociales y culturales.

Bourhis, R. Y., L. C. Moise, S. Perreault and S. Senécal. 1997. "Towards an interactive acculturation model: A social psychological approach". International Journal of Psychology 32:369-386. http://dx.doi.org/10.1080/002075997400629

Briones, E., M. Verkuyten, J. Cosano and C. Tabernero. 2012. "Psychological adaptation of Maroccan and Ecuadorean immigrant adolescents in Spain". International Journal of Psychology 47:28-38. http:// dx.doi.org/10.1080/00207594.2011.569722

Brown, R. 1995. Prejudice: its social psychology. Oxford, UK: Blackwell.

Chen, G. M. 1990. "Intercultural communication competence: Some perspectives of research". The Howard Journal of Communications 2:243-261. http://dx.doi. org/10.1080/10646179009359718

Chen, G. M. 1997. "A Review of the Concept of Intercultural Sensitivity". Paper presented at the Biennial Convention of the Pacific and Asian Communication Association, Honolulu, HI.

Chen, G. M. and L. C. Starosta. 1996. "Intercultural communication competence: A synthesis". In B. R. Burleson (Ed.), Communication Yearbook 19:353-384.

Chen, G. M. and W. J. Starosta. 2000. "The development and validation of the intercultural communication sensitivity scale". Human Communication 3:1-15. 
Chen, G. M. and W. J. Starosta. 2004. "Communication among cultural diversities: A dialogue". International and Intercultural Communication Annual 27:3-16. http:/l dx.doi.org/10.4135/9781452229720

Gudykunst, W. B., Wiseman, R. L. and Hammer, M. R. 1977. "An analysis of an integrated approach to cross-cultural training". International Journal of Intercultural Relations 1:99110. http://dx.doi.org/10.1016/0147-1767(77)90045-1

International Organization for Migration. 2009. About migration. Retrieved April 18, 2009 (http://www.iom.int/jahia/ Jahia/about-migration/lang/en).

Kosic, A., L. Mannetti and D. L. Sam. 2005. "The role of majority attitudes towards out-group in the perception of the acculturation strategies of immigrants". International Journal of Intercultural Relations 29:273-288. http:// dx.doi.org/10.1016/j.jijintrel.2005.06.004

Navas, M., M. C. García, A. Rojas, P. Pumares and I. Cuadrado. 2006. "Actitudes de aculturación y prejuicio: la perspectiva de autóctonos e inmigrantes". Psicothema 18:187-193.

Navas, M., M. C. García, J. Sánchez, A. J. Rojas, P. Pumares and J. S. Fernández. 2005. "Relative acculturation extended model (RAEM): New contributions with regard to the study of acculturation". International Journal of Intercultural Relations 29:21-37. http://dx.doi. org/10.1016/j.ijintrel.2005.04.001

Navas, M., A. J. Rojas and M. C. García. 2011. "Concordancia entre actitudes y percepciones de aculturación de la población autóctona hacia los inmigrantes magrebíes: relación con las actitudes prejuiciosas". Anales de Psicología 27:186-194.

Navas, M., A. J. Rojas, M. C. García and P. Pumares. 2007. "Acculturation strategies and attitudes according to the Relative Acculturation Extended Model (RAEM): The perspectives of natives versus immigrants". International Journal of Intercultural Relations 31:6786. http://dx.doi.org/10.1016/j.ijintrel.2006.08.002

Padilla, A. M. 1980. "The role of cultural awareness and ethnic loyalty in acculturation". Pp 47-84 in Acculturation: Theory, models and some new findings, edited by A. M. Padilla Boulder. Colorado: Westview Press.

Padilla, A. M. and W. Perez. 2003. "Acculturation, Social Identity, and Social Cognition: A New Perspective". Hispanic Journal of Behavioral Sciences 25:35-55. http://dx.doi.org/10.1177/0739986303251694

Pfafferott, I. and R. Brown, R. 2006. "Acculturation preferences of majority and minority adolescents in Germany in the context of society and family". International Journal of Intercultural Relations 30:703-717. http://dx.doi. org/10.1016/j.jijintrel.2006.03.005

Piontkowski, U., A. Rohmann and A. Florack. 2002. "Concordance of acculturation attitudes and perceived threat". Group Processes \& Intergroup Relations 5:221 232. http://dx.doi.org/10.1177/1368430202005003003

ANTONIO GONZÁLEZ LÓPEZ es doctor en Psicología Social por la Universidad Complutense de Madrid (España). Durante los últimos años ha compaginado su labor profesionalen los Servicios Sociales con actividad académica como profesor asociado en la Universidad de Castilla La Mancha y como profesor tutor de la UNED en Cuenca. Sus áreas de interés investigador han sido la preocupación ambiental, las TIC en mayores y actualmente las actitudes de aculturación.
Portes, A., A. Celaya, E. Vickstrom, and R. Aparicio. 2012. "Who are we? Parental Influences on Self-identities and Self-esteem on Second Generation Youths in Spain". Revista Internacional de Sociología (RIS) 70:937. http://dx.doi.org/10.3989/ris.2011.09.26

Redfield, R., R. Linton and M. J. Herskovits. 1936. "Memorandum on the study of acculturation". American Anthropology 38:149-152. http://dx.doi.org/10.1525/ aa.1936.38.1.02a00330

Rodríguez-García, D. 2010. "Beyond assimilation and multiculturalism: a critical review of the debate on managing diversity". International Migration and Integration 11:251271. http://dx.doi.org/10.1007/s12134-010-0140-x

Rojas, A. J., M. Navas, O. M. Lozano and P. J. Pérez. 2011. "Prejudiced attitude measurement using the rasch rating scale model". Psychological Reports 109:553-572. http://dx.doi.org/10.2466/07.17.PR0.109.5.553-572

Ruben, B. D. and D. J. Kealy. 1979. "Behavioural assessment of communication competency and the prediction of cross-cultural adaptation". International Journal of Intercultural Relations 3:15-48. http://dx.doi. org/10.1016/0147-1767(79)90045-2

Rudmin, F. W. 2009. "Constructs, measurements and models of acculturation and acculturative stress". International Journal of Intercultural Relations 33:106-123. http:// dx.doi.org/10.1016/j.ijintrel.2008.12.001

Ryder, A. G., L. E. Alden and D. L. Paulhus. 2000. "Is Acculturation Unidimensional or Bidimensional? A Head-to-Head Comparison in the Prediction of Personality, Self-identity, and Adjustment". Journal of Personality and Social Psychology 79:49-65. http:// dx.doi.org/10.1037/0022-3514.79.1.49

Van Oudenhoven, J. P., K. S. Prins and B. P. Buunk. 1998. "Attitudes of minority and majority members toward adaptation of immigrants". European Journal of Social Psychology 2:995-1013. http://dx.doi.org/10.1002/ (SICI)1099-0992(1998110)28:6<995::AID EJSP908>3.0.CO;2-8

Ward, C. and A. Kennedy. 1994. "Acculturation strategies, psychological adjustment, and sociocultural competence during cross-cultural transitions". International Journal of Intercultural Relations 18:329-343. http://dx.doi. org/10.1016/0147-1767(94)90036-1

Zagefka, H., and R. Brown. 2002. "The relationship between acculturation strategies, relative fit and intergroup relations: Immigrant-majority relations in Germany". European Journal of Social Psychology 32:171-188. http://dx.doi.org/10.1002/ejsp.73

Zick, A., U. Wagner, R. Van Dick and T. Petzel. 2001. "Acculturation and prejudice in Germany: Majority and minority perspectives". Journal of Social Issues 57:541557. http://dx.doi.org/10.1111/0022-4537.00228

PAZ RAMÍREZ LÓPEZ es diplomada en Trabajo Social, Máster en Migraciones Internacionales y Doctoranda por la Universidad Pontificia Comillas Madrid (España). Su Proyecto de Investigación de Tesis Doctoral trata sobre la aculturación psicosocial de la población inmigrante. Durante los últimos años ha compaginado su profesión en Cáritas Cuenca con sus estudios de Máster y Doctorado. Sus líneas de investigación han sido las TIC en personas mayores y la aculturación psicosocial de los inmigrantes. 